quarumdam conicarum sectionum. Pleroides torricellianae theorema mecum demonstrant, ideoque ipsum hic minime praeterire decebat: „ passa quindi alla dimostrazione. Da quanto si è di sopra esposto resta fuor di dubbio che i lavori del Casali siano restati sconosciuti dai Geometri moderni, che si sono occupati delle proprietà della logociclica; d'altronde il Sig. Booth nelle sue ricerche non ha avulo occasione di riconoscere la curva come una focale regolare. Gli eruditi cultori della geometria potrebbero rintracciare ed indicare in quali Opere del Torricelli siano stale prese ad esame queste curve che dal Grandi, che giusta le parole del Casali, vengono sempre chiamate Pteroides Torricellianae. Terminerò quest'articolo col nolare che nella citata collezione dei Commentarii Instituti Bononiensis si trovano altre Memorie del Casali, di geometria, di algebra, e di Fisica meccanica : piacemi di richiamarne l'ultima di geometria, ed impressa nel tomo $7^{\circ}$ 1791: così a pag. 338 si legge il seguente titolo: Gregorii Philippi Mariae Casalii Bentivoli Paleotti. De polygonoidum area. Questo articolo contiene nove pagine con una tavola di figure. Il Casali vuol ritrovare la superficie generata da un poligono che con moto di rolazione si ravvolge sopra una retta : per il poligono regolare a pag. 340 enuncia, e dimostra il seguente teorema " Si F aream regularis figure denotet, quae polygonoidem generat, $c$ vero aream circuli eidem figurae circumscripti, dico aream polygonoidis esse $=\mathrm{F}+2 c$. "Per la cicloide si trova subito esser l'area della curva tripla del circolo generatore. Il Casali apparteneva a nobile famiglia, congiunto egualmente ad altre nobili famiglie come lo indicano i suoi cognomi.

Roma 30 Maggio 1861.

Barnaba Tortolini.

\title{
PUBBLICAZIONI RECENTI
}

Piuma Carlo Maria. - Nola sulla determinazione della parte algebrica nell'integrazione in funzione finita esplicita. Fasc. in . 4. di pag. 16. Genova 1860.

Cremona D. Luigi - Memoria sulle superficie gubbe del terz'ordine. Fasc. in $4^{\circ}$ di pag. 14. Milano 1861. (Estratta dall'Istituto Lombardo vol. II.)

Staud - Beiträge zur geometrie der lage $3^{e}$. Heft (Schlussheft). Nürnberg 1860.

Bserknes, Über die geometrische Repräsentation der g'leichungen swischeu zwei veränderlichen reellen oder komplexen Grössen, Christiania $1859^{\circ}$ (Universtäts program für das zweite Halljahr 1859).

Clebsch, Ueber Symbolische Dar Stellung algebraischer Formen (Giornale Matematico di Berlino (om. 59). 